\title{
dc septum magnet based on permanent magnet for next-generation light sources
}

\author{
Tsutomu Taniuchi®, ${ }^{1, *}$ Takahiro Watanabe $\odot,{ }^{1,2}$ Shiro Takano $\odot,{ }^{1,2}$ Tsuyoshi Aoki, ${ }^{1}$ \\ Kenji Fukami, ${ }^{1,2}$ Shinichi Matsubara $\odot,{ }^{1}$ Kenichi Yanagida, ${ }^{1}$ Kazuhiro Hamato, ${ }^{3}$ Jun Kataoka, ${ }^{3}$ \\ Kanichiro Ogata, ${ }^{3}$ Yoshiyuki Saito, ${ }^{3}$ and Kunihiro Kusano ${ }^{3}$ \\ ${ }^{1}$ Japan Synchrotron Radiation Research Institute (JASRI), 1-1-1 Kouto, Sayo, Hyogo 679-5198, Japan \\ ${ }^{2}$ RIKEN SPring-8 Center (RSC), 1-1-1 Kouto, Sayo, Hyogo 679-5148, Japan \\ ${ }^{3}$ TOKIN Corporation, 6-7-1 Koriyama, Taihaku, Sendai 982-0003, Japan
}

(Received 11 October 2019; published 2 January 2020)

\begin{abstract}
This paper presents a dc septum magnet based on a permanent magnet that we developed as an injection magnet for next-generation light sources. Permanent-magnet based magnet devices are regarded as one of the key technologies for next-generation light sources as they are expected to consume less power, occupy less space, and cause fewer failures than conventional electromagnets that require power sources. The injection magnets, including the dc septum magnet, are often restricted to a limited space, and consume large amounts of power. Thus, the advantage of building such a magnet using a permanent magnet should be as important as that of building main magnets. We designed and fabricated a permanent-magnet based dc septum magnet with a thin septum plate of $7 \mathrm{~mm}$ thickness, and confirmed that the measured gap and leakage fields agreed well with the design values. The permanent-magnet specific issues such as a variablefield mechanism, demagnetization and temperature dependence, are also solved. Furthermore, we explored the numerical study for reducing the septum thickness, and verified that the double-septum structure enabled a reduction in the overall septum thickness, while keeping adequate magnetic shielding.
\end{abstract}

DOI: 10.1103/PhysRevAccelBeams.23.012401

\section{INTRODUCTION}

One of the new features of next-generation light sources compared with the third generation ones is the use of permanent magnets (PMs) for the magnets in their magnetic lattice, such as bending magnets of a storage ring [1-3]. A PM consumes less power than electromagnets, and its compactness due to a lack of coils can be beneficial in cases where the packing factor is high. Moreover, accidental beam trips due to power supply failures are expected to be reduced significantly because these magnets do not require any electric feed. These features may match requirements for future light sources, where the lattice consists of many strong magnets. Furthermore, stability and reliability are important factors for user facilities. In fact, a variety of magnets based on the PM have been proposed, developed, and evaluated [4-11].

At SPring-8, we designed and fabricated several types of PM-based dipole magnets, such as the longitudinal gradient

\footnotetext{
*ihcuinat@spring8.or.jp

Published by the American Physical Society under the terms of the Creative Commons Attribution 4.0 International license. Further distribution of this work must maintain attribution to the author(s) and the published article's title, journal citation, and DOI.
}

and combined-function bending magnets, as well as normal bending magnets [12]. In these studies, we proposed what we call outer and inner plates for smoothly tuning the magnetic flux density on the beam axis, and also introduced $\mathrm{Fe}-\mathrm{Ni}$ alloys for compensating the temperature dependence of the PM. To prevent demagnetization due to radiation exposure, a samarium-cobalt $\left(\mathrm{Sm}_{2} \mathrm{Co}_{17}\right)$ magnet was chosen and the permeance coefficient was set as high as possible. In the present work, we designed and fabricated a PM-based septum magnet, which should act as a functional substitute for a dc septum electromagnet in the beam injection part of future light sources.

A new off-axis beam injection scheme, in-vacuum transparent off-axis beam injection [13], proposed for the SPring-8 upgrade, SPring-8-II [1], is shown in Fig. 1. A PM-based dc septum magnet was chosen considering its spatial constraint and other advantages compared with electromagnet systems. The PM-based dc septum magnet is expected to provide a stable magnetic field free of power supply output variations and enables the stable beam deflection necessary for the small injected beam oscillation amplitude, for both stacking and maintaining, by toppingup the required beam intensity.

The challenge of developing a PM-based dc septum magnet is to have a strong magnetic field that deflects the incident beam and minimize the leakage magnetic field for 


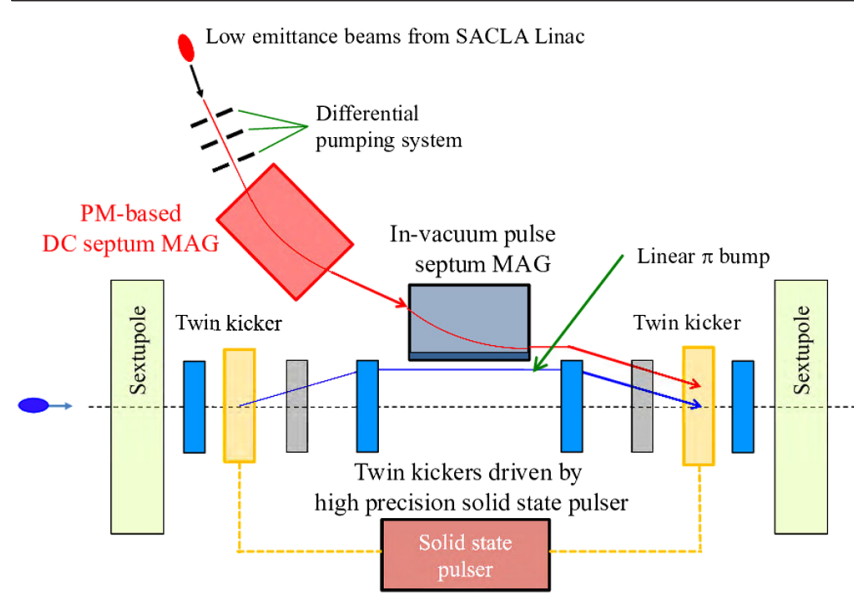

FIG. 1. Proposed injector section of SPring-8-II [13].

the transparent beam injection in a compact size. Therefore, the leakage magnetic field emanating from the septum should be suppressed to as low as possible, so that stored beam does not experience it. Note that the leakage magnetic field is constant in time, but still needs to be suppressed because the stored beam may be kicked by the leakage field in different ways depending on the bump amplitude while the kicker magnets are making the bump. For the nextgeneration light sources, the dynamic aperture is naturally narrower than those for the third generation ones due to its strong chromatic and geometric aberrations in beam optics. Thus, the septum magnets need to be designed in such a way that the distance between the deflecting field excited for the incident beam and nonfield area out of the septum for the stored beam is kept as close as possible. Especially for the dc-type septum magnet, the leakage magnetic field needs to be suppressed without the help of eddy current.

The main parameters of the dc septum are summarized in Table I. The deflecting field is set to be $1.2 \mathrm{~T}$ so that the incident beam is kicked by the field $24 \mathrm{mrad}$ for the electron energy of $6 \mathrm{GeV}$. In the following, the magnet design parameters are defined according to the magnet configuration in Fig. 1, but in the paper we present general discussion on the design and fabrication of a permanent magnet-based dc magnet.

\section{DESIGN AND FABRICATION}

To design the magnetic circuit of the PM-based septum magnet, we considered the leakage field reduction, margin for the demagnetization due to radiation, variable range of

TABLE I. Main parameters of de septum magnet.

\begin{tabular}{lc}
\hline \hline Magnetic flux density at pole gap (T) & 1.2 \\
Gap length between poles (mm) & 10 \\
Longitudinal pole length (mm) & 400 \\
Kick angle @ 6 GeV (mrad) & 24 \\
\hline \hline
\end{tabular}

the gap field, reduction of the temperature coefficient, monitoring for the long-term change of the field strength, and so on. We present the details regarding these factors in the following sections.

\section{A. Selection of PM material}

For the actual magnetic circuit design, we chose a rare earth magnet material from two candidates, $\mathrm{Nd}-\mathrm{Fe}-\mathrm{B}$ and $\mathrm{Sm}_{2} \mathrm{Co}_{17}$, both of which have high residual magnetic flux density (remanence) $B_{r}$ to produce a necessary gap field and high coercivity, $\mathrm{H}_{c}$, which relates to the resistance against demagnetization. Nd-Fe-B has a higher $\mathrm{B}_{\mathrm{r}}$ and $\mathrm{H}_{\mathrm{c}}$ than $\mathrm{Sm}_{2} \mathrm{Co}_{17}$, and the total number of PMs can be reduced. However, we chose one of the highest grades of commercially available $\mathrm{Sm}_{2} \mathrm{Co}_{17}$ (LM-32SH, TOKIN Corporation [14]) considering its toughness against demagnetization in an irradiated environment [15]. The specification of $\mathrm{Sm}_{2} \mathrm{Co}_{17}$ adopted for the septum magnet is summarized in Table II.

\section{B. Magnetic circuit layout}

It is important to choose the operating point of the PM on the $\mathrm{B}-\mathrm{H}$ curve or demagnetization curve in a magnetic circuit design. The operating point can be expressed as a permeance coefficient, $P_{c}$, which is used to design PM applications and is defined as the ratio of flux density $B_{d}$ and magnetic field $H_{d}$ at the operating point as follows.

$$
P_{c}=-\frac{1}{\mu_{0}} \frac{B_{d}}{H_{d}} .
$$

Note that $B_{d}$ and $H_{d}$ are components of vector fields in the direction of magnetization of the PM. The higher the $P_{c}$, the lower the inverse magnetic field in the PM, and the higher the margin against demagnetization due to radiation. This is similar to the temperature rise process as shown in Fig. 2.

The layout of PMs was determined considering $P_{c}$, the magnetic saturation in pole pieces, the total number of PMs reflecting the cost, manufacturing process, etc.

The septum magnet was designed with a simulation code CST Studio Suite [16]. Figure 3 shows the schematic view of the designed septum magnet. In this figure, the PMs producing a magnetic flux for deflecting beam are located in the vertical and horizontal positions of the pole piece.

TABLE II. Specification of $\mathrm{Sm}_{2} \mathrm{Co}_{17}$ [14].

\begin{tabular}{lc}
\hline \hline Remanence, $\mathrm{B}_{\mathrm{r}}(\mathrm{T})$ & $1.12-1.2$ \\
Coercivity, $\mathrm{H}_{\mathrm{cB}}(\mathrm{kA} / \mathrm{m})$ & $795-875$ \\
Coercivity, $\mathrm{H}_{\mathrm{cJ}}(\mathrm{kA} / \mathrm{m})$ & $>1592$ \\
Maximum energy product $\left(\mathrm{kJ} / \mathrm{m}^{3}\right)$ & $223-256$ \\
Temperature coefficient, $\mathrm{T}_{\mathrm{c}}\left(\% /{ }^{\circ} \mathrm{C}\right)$ & -0.04 \\
\hline \hline
\end{tabular}




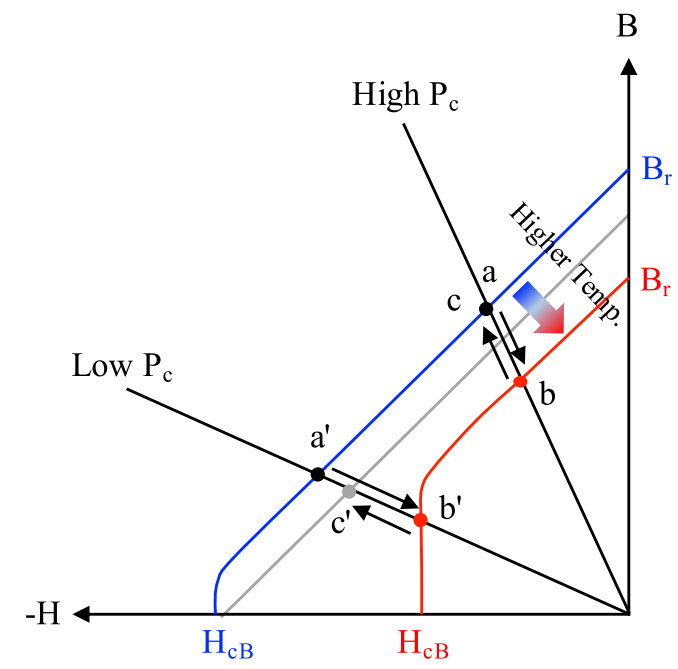

FIG. 2. Movement of operating point on B-H curve under heat cycle. While the operating point moves a-b-c for high $P_{c}$, it moves a'-b'-c' for low $P_{c}$, and an irreversible demagnetization occurs.

Additional PMs are installed between the pole piece and a septum plate for cancelling out the fringe field. In order to protect these magnets from demagnetization, especially those close to the beam axis, each magnet piece is designed to be thick enough to have a large $P_{c}$. Magnetic-shunt alloy plates for temperature compensation and a movable shunt plate for varying the field strength are located close to side PMs. Pole pieces were made of pure iron and return yokes and plates were JIS-SS400-specified steel [17].

The simulated distributions of the magnetic flux density (B) the magnetic field $(\mathrm{H})$ at the longitudinal center of the magnetic circuit are shown in Fig. 4 and Fig. 5, respectively.

With the $\mathrm{B}$ and $\mathrm{H}$ data, $P_{c}$ was calculated from Eq. (1). Figure 6 shows the distribution of $P_{c}$ in PMs. The average values of $P_{c}$ in each PM are also shown in the figure.

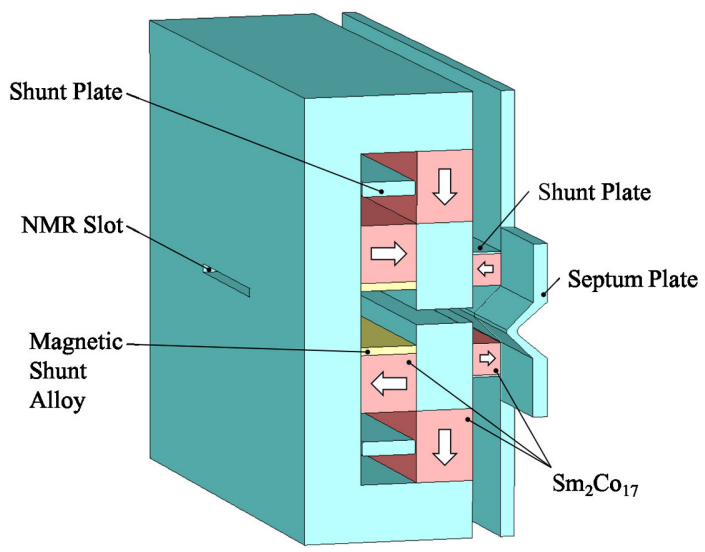

FIG. 3. Layout of magnetic circuit components.
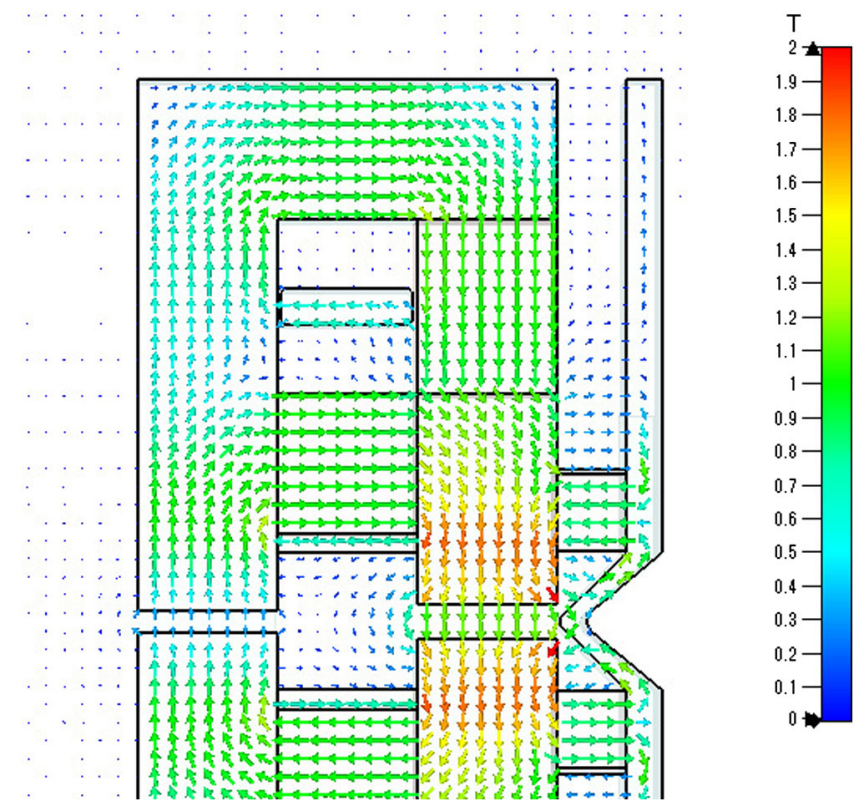

FIG. 4. Magnetic flux density (B) distribution at the central cross section in the beam direction.

\section{Septum plate}

On the one hand, it is desirable to have the incident beam and stored beam closer to each other, to reduce the field strength of a pulsed septum magnet in the downstream. On the other hand, the leakage field from the gap must be sufficiently low to avoid any perturbations to the stored beam. As the dipole field is constant with time, an eddy current, which can suppress the leakage field in case of a pulsed magnet, is not excited in the septum. Therefore, the septum and the additional magnetic shield plates, if necessary, shield the magnetic field by their high magnetic permeabilities compared to the ambient air. A thin septum

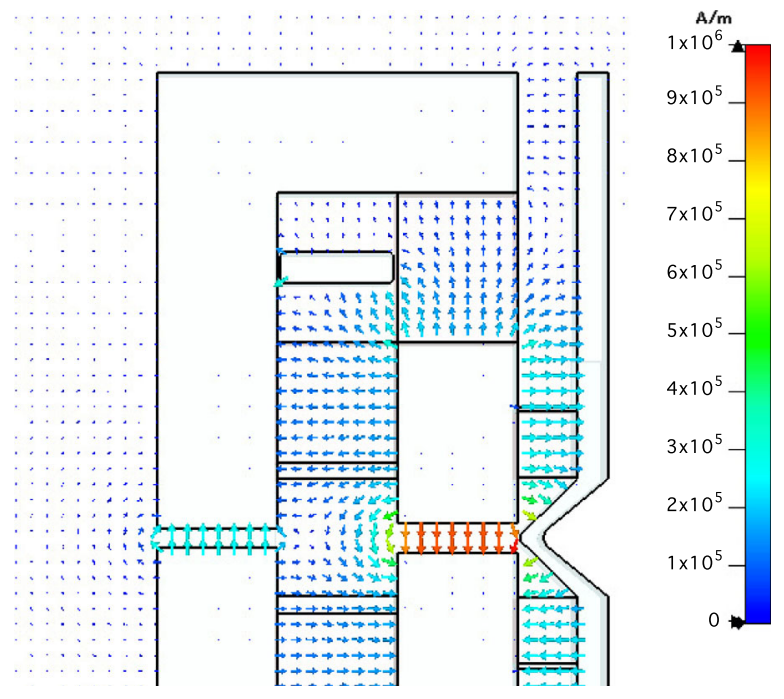

FIG. 5. Magnetic field $(\mathrm{H})$ distribution at the central cross section in the beam direction. 


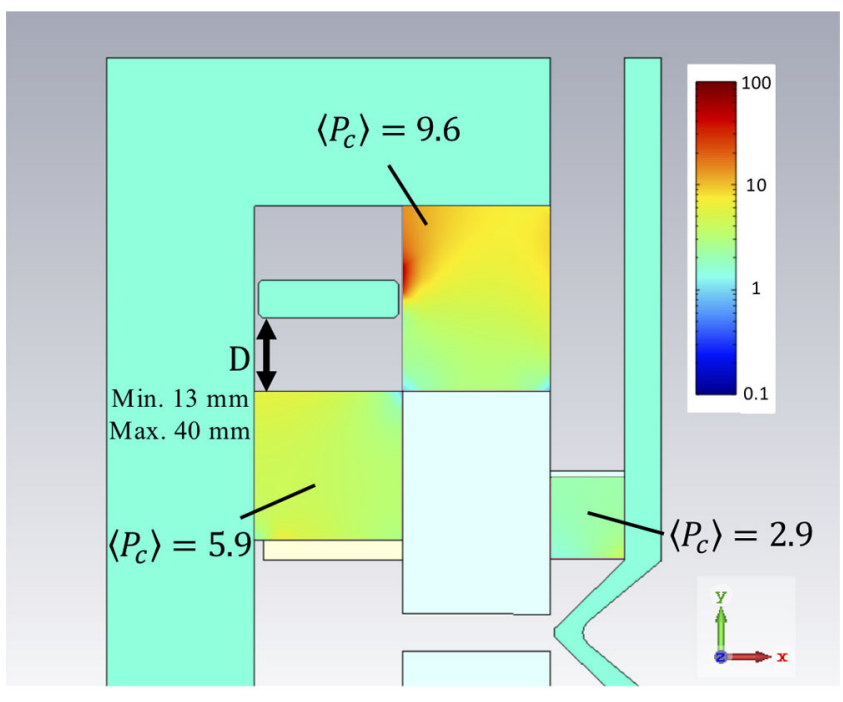

FIG. 6. Permeance coefficient $\left(P_{c}\right)$ distribution in PMs for the shunt plate position (D) of $20 \mathrm{~mm} .\left\langle P_{c}\right\rangle$ s are the average value in PMs.

plate and a short distance between the septum plate and the pole piece can bring closer the orbits of the incident and stored beams. However, magnetic saturation in the septum plate becomes higher and the leakage field from the septum plate increases.

Therefore, we adopted a counter PM to cancel the magnetic flux in the septum plate. This made the plate thinner and we achieved the thickness of $7 \mathrm{~mm}$ on the median plane. Such a technique has been employed for the end-field termination in undulator magnet arrays [18]. Furthermore, the septum plate was bent in a wedgeshape to reduce the distance between the incident and stored beam orbits.

The leakage field can be minimized by adjusting a counter flux in the septum plate. Steel plates with a thickness of $1 \mathrm{~mm}$ each were stacked near the counter PM, to shunt the magnetic flux and adjust the counter flux. The thickness was adjusted to minimize the leakage field while measuring the magnetic field outside the septum plate.

The fringe field in the longitudinal direction, especially at the pole end near the injection point, must also be cured. The length of the septum plate was increased by $80 \mathrm{~mm}$ in the longitudinal direction to shield the fringe field from the pole end, as can be seen in Fig. 7.

\section{Variable-field mechanism}

In PM circuit manufacturing, it is rather difficult to optimize the gap field to a design value within a reasonable cost. One of the conventional ways is shimming. In our case, the so-called outer plate mechanism was developed for changing the gap field smoothly and quickly [12]. However, as shown in Fig. 4, a shunt plates moving inside the return yoke was adopted to the septum magnet

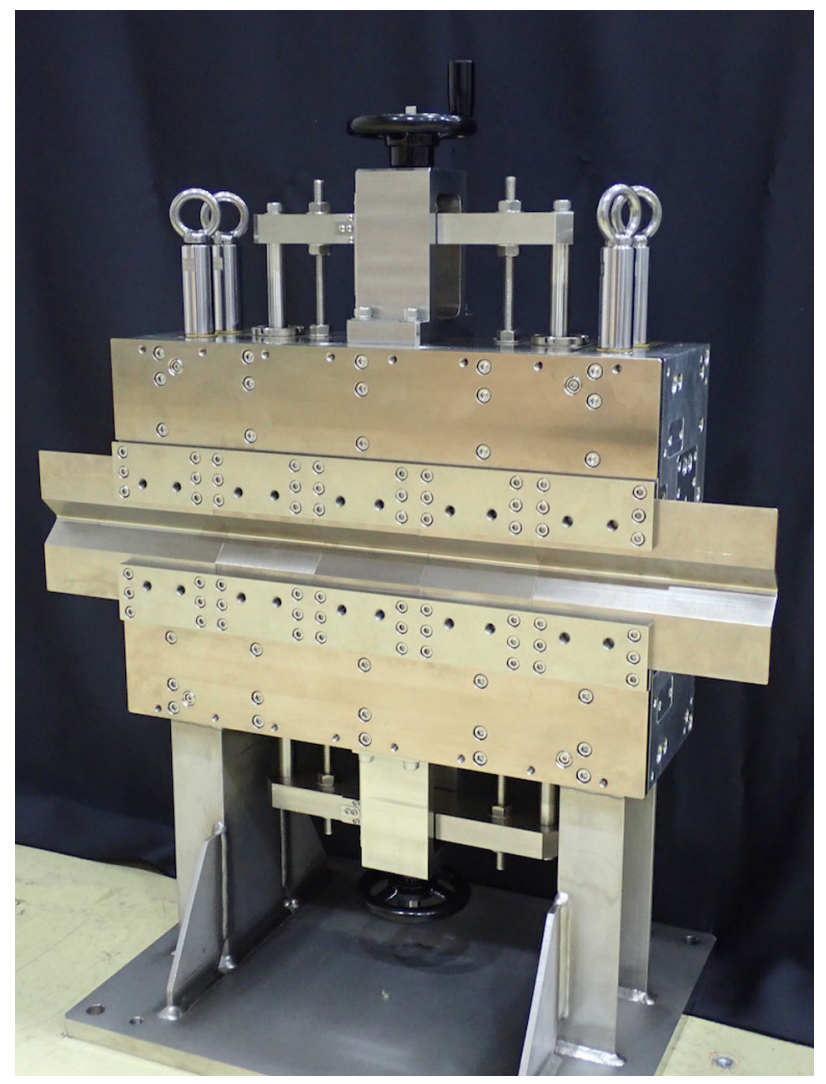

FIG. 7. Fabricated PM-based septum magnet. Septum plates are extended by $80 \mathrm{~mm}$ to the shield fringe field in the beam direction.

considering the compactness required by a dense injection section for SPring-8-II. Shunt plates can be moved vertically using rotating handles attached on top and bottom of the magnet. As the shunt plate gets closer to the PM, the magnetic flux in the plate increases and the gap-field strength decreases. Components of the bearing system were chosen to counter the magnetic force acting on the shunt plates shown in Fig. 8.

The variable range of the gap field was designed to be $3 \%$ considering a fabrication error and recovery from timedepending demagnetization.

\section{E. Field monitoring slot}

To guarantee the beam trajectory during actual machine operation, the magnetic field strength at the pole gap must be monitored. Whereas, the gap field strength can be monitored indirectly by monitoring the excitation current in the electromagnet, the magnetic field strength itself must be monitored using field measuring devices such as a Hall sensor or a nuclear magnetic resonance (NMR) Teslameter. However, it is difficult to insert these sensors into the small gap of the septum magnet, which is occupied by the vacuum chamber, and widening the pole pieces leads to an increase in the PMs weight and cost. Instead, we 


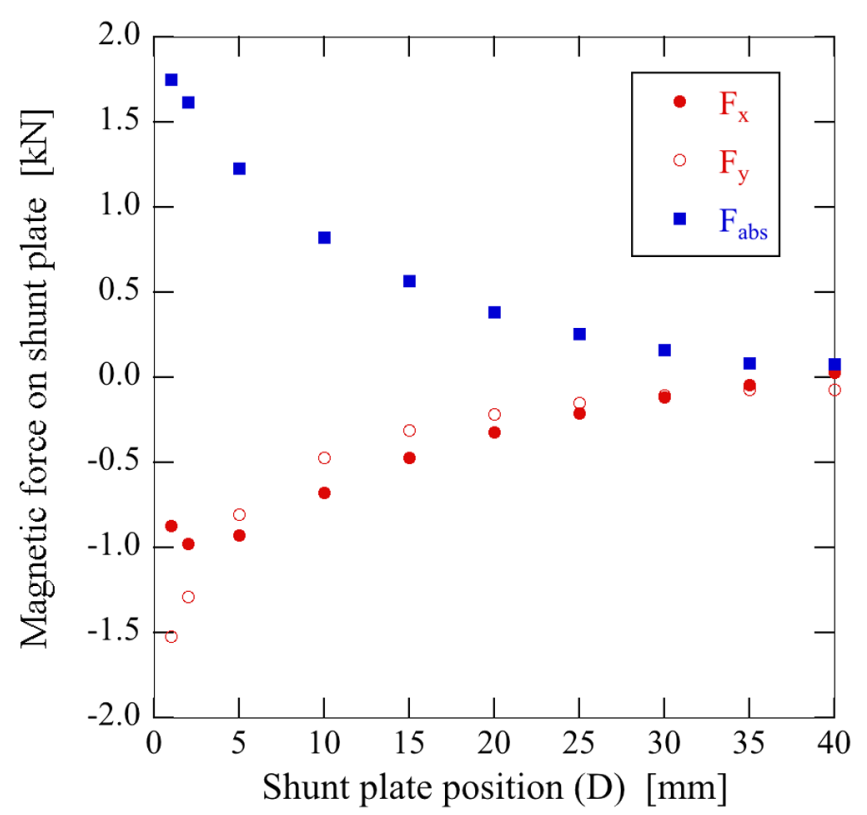

FIG. 8. Simulated magnetic forces acting on a tuning shunt plate as a function of the shunt plate position (D), where $F_{x}$ and $F_{y}$ are the horizontal and vertical components, respectively. (The coordinate system is shown in Fig. 6). The movable range of the plate in the fabricated septum magnet is $13-40 \mathrm{~mm}$.

adopted a slot in the return yoke to monitor the field strength correlated to one at the pole gap. Figure 9 shows the correlation between field strengths at the pole gap and the return-yoke slot (NMR slot). The field strength in the NMR slot depends on the magnetic saturation of the yoke near that slot. We chose a proper thickness of the return yoke and a slot size to induce a sufficient magnetic field strength into the slot.

The NMR Teslameter was chosen for the field monitoring owing to its higher resolution $\left(<10^{-6}\right)$, lower

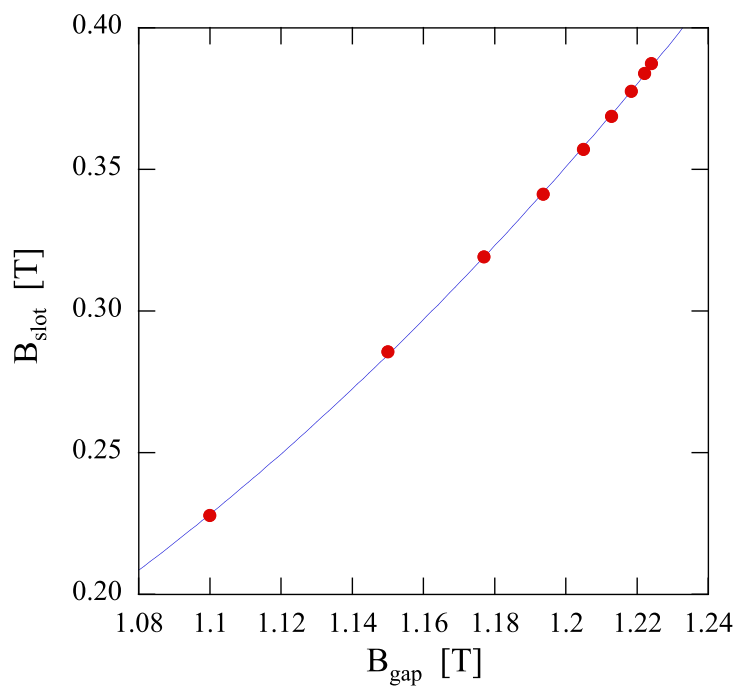

FIG. 9. Correlation between field strengths at the pole gap and return-yoke slot.

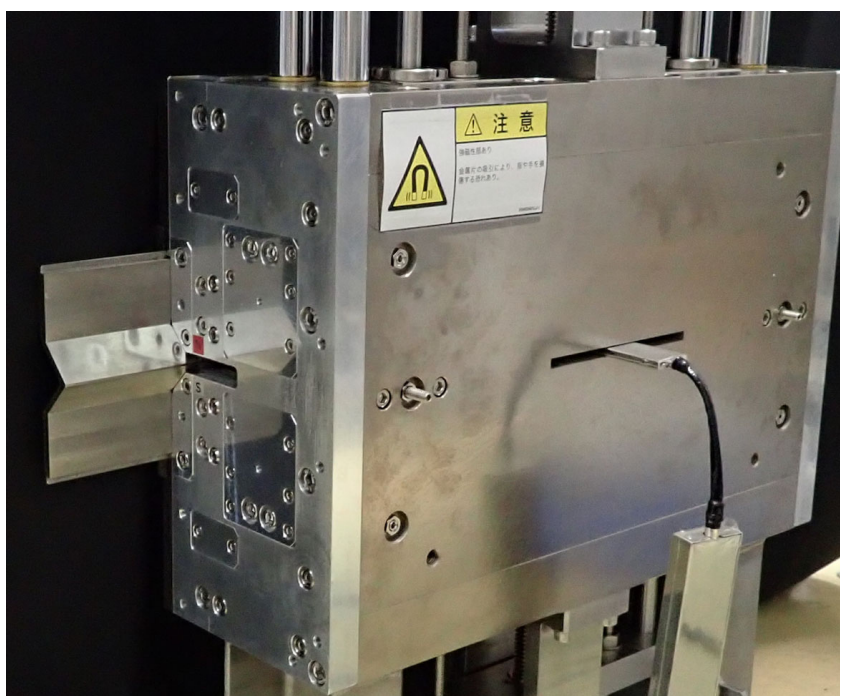

FIG. 10. Field monitoring slot at the return-yoke and inserted NMR probe.

temperature coefficient $\left(10^{-7} /{ }^{\circ} \mathrm{C}\right)$, and resistance to radiation compared to Hall sensors. The dimensions of the field detecting volume in the NMR probe are designed to be $5 \mathrm{~mm}(\mathrm{H}) \times 15 \mathrm{~mm}(\mathrm{~W}) \times 5 \mathrm{~mm}(\mathrm{D})$ for installing in a slot of height $6 \mathrm{~mm}$. In this volume, a field uniformity of $10^{-4}$ is required to obtain a good signal level for the NMR waveform. Figure 10 shows the NMR probe installed in the monitoring slot.

\section{F. Compensation for temperature dependence}

The temperature coefficient $\left(\mathrm{T}_{\mathrm{c}}\right)$ of $\mathrm{Sm}_{2} \mathrm{Co}_{17}$ is typically $-0.04 \% /{ }^{\circ} \mathrm{C}$. Although it is smaller than that of Nd-Fe-B, which is typically $-0.1 \% /{ }^{\circ} \mathrm{C}$, it may not be negligible for a stable beam operation. We have demonstrated that the temperature dependence of the gap field strength in PMbased dipoles can be reduced sufficiently using an Fe-Ni alloy (magnetic-shunt alloy) [12]. The same technique was applied for this septum magnet. We set the target range of the temperature coefficient to $\pm 5 \times 10^{-5} /{ }^{\circ} \mathrm{C}$. The magnetic-shunt alloy used as a temperature compensator was MS-2 [19], in which the composition ratio of $\mathrm{Ni}$ was $31 \%$ and the linearity of temperature dependence was optimized for the temperature range from 20 to $40^{\circ} \mathrm{C}$.

We measured the $\mathrm{B}-\mathrm{H}$ curve of the alloy at magnetic fields of up to $80 \mathrm{kA} / \mathrm{m}$. Using these data in the simulation, a necessary amount of MS-2 was estimated to minimize the $T_{c}$. The magnetic-shunt alloy was rolled into 1-mmthick plates, which were stacked near the PMs. The total thickness of MS-2 was adjusted while measuring the $T_{c}$ by using an NMR Teslameter (Echo-Denshi KK) in a temperature-controlled booth. Figure 11 shows the history of the MS-2 thickness optimization. The optimal thickness was $5.5 \mathrm{~mm}$; an alloy plate was cut in half to be equivalent to the thickness of $0.5 \mathrm{~mm}$. The temperature dependence at a 


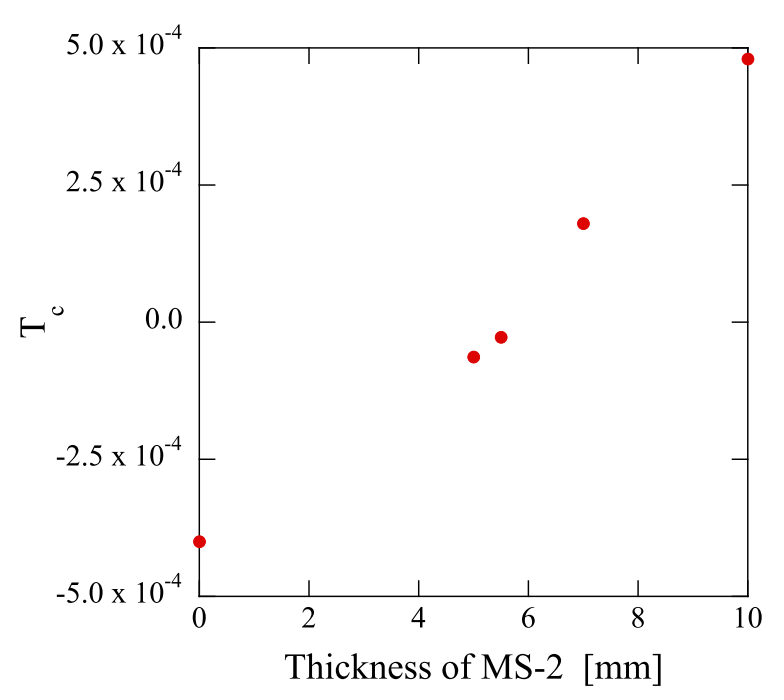

FIG. 11. Temperature coefficient minimization through optimization of magnetic-shunt alloy (MS-2) thickness.

thickness of $5.5 \mathrm{~mm}$ is shown in Fig. 12. $\mathrm{T}_{\mathrm{c}}$ was expected to be sufficiently small around an atmospheric temperature of $27^{\circ} \mathrm{C}$ in the accelerator tunnel at SPring-8.

\section{G. Fabrication}

The fabrication process of the septum magnet consists of PM block production, iron yoke, frame fabrication, and assembly.

In PM block production, basic PM pieces were sintered and shaped. They were then, using a heat-resistant adhesive, bonded to form PM blocks for the magnetization. In order to reduce the initial time-dependent demagnetization, the PM blocks were baked [20] at a temperature of $200{ }^{\circ} \mathrm{C}$.

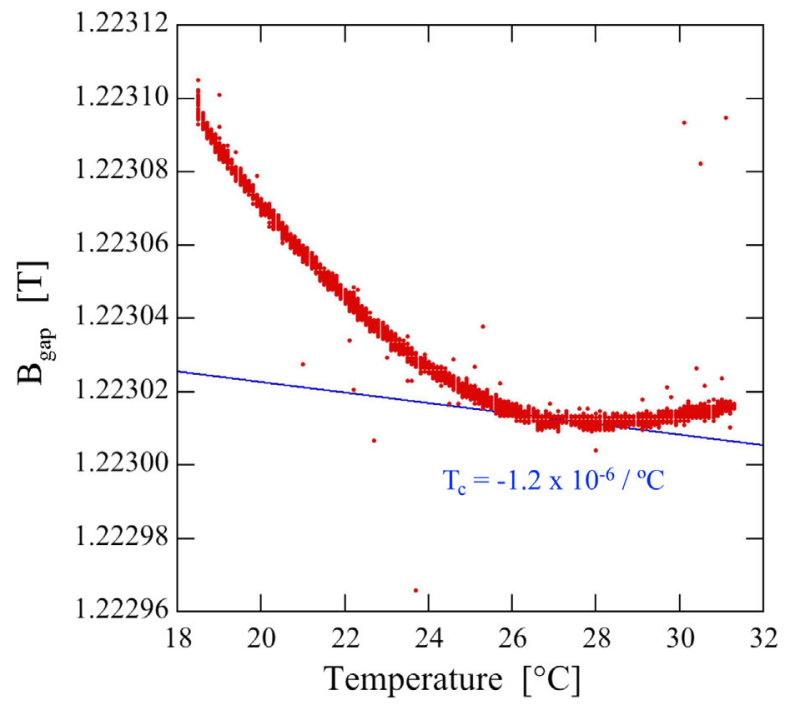

FIG. 12. Temperature dependence of gap field after MS-2 thickness optimization. The value of $\mathrm{T}_{\mathrm{c}}$ was obtained by fitting data in the range of $26-28^{\circ} \mathrm{C}$.
The typical demagnetization at a PM surface after the baking was $0.5-1 \%$.

The septum magnet was assembled in a booth to prevent contamination from magnetic dust. The strong magnetic field zone was specified by the booth for safety. A device to alert against strong magnetic fields was worn by workers in the booth to prevent unintended exposure to the strong field.

To overcome the strong magnetic force, the PMs using an assembly jig were introduced into iron yokes. The gap distance was required to be precisely aligned to ensure a good field region. We adopted nonmagnetic side frames made of aluminum to position the pole pieces within a required tolerance and support them against the attractive magnetic force, and installed them at both ends of the pole pieces.

\section{MAGNETIC FIELD MEASUREMENT}

\section{A. Gap-field variable range}

As described in preceding section, the gap field strength can be varied by moving the shunt plates inside the return yoke. Figure 13 shows the dependence of the measured gap-field strength on the upper and lower shunt plate positions. In this case, the lower plate was moved from $\mathrm{D}=13$ to $40 \mathrm{~mm}$ while the upper plate was fixed at $\mathrm{D}=13 \mathrm{~mm}$; then, the upper plate was moved from $\mathrm{D}=13$ to $40 \mathrm{~mm}$ while the lower plate was fixed at $\mathrm{D}=40 \mathrm{~mm}$. The variable range of approximately $2 \%$ is obtained by moving one of the upper or lower plates, and in total, a variable range of $3.9 \%$ is ensured by moving the both plates.

When the upper and lower plates are in asymmetrical positions, the magnetic field distribution in the gap may be slightly deformed. According to our numerical simulation

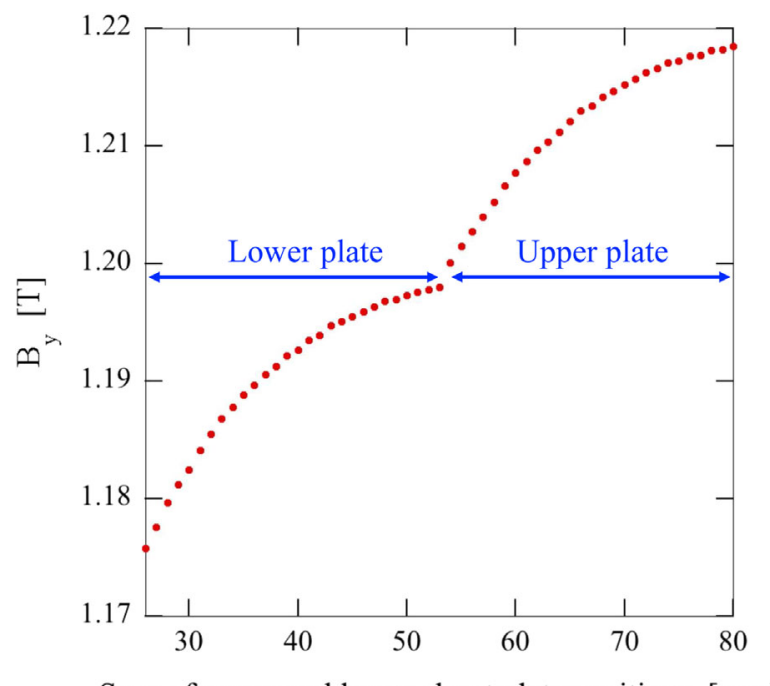

Sum of upper and lower shunt plate positions [mm]

FIG. 13. Gap-field strength variation measured by moving the lower and upper shunt plates. 
for Fig. 13, the dipole field is deformed in the vertical axis by $d B_{y} / d y=0.2 \mathrm{~T} / \mathrm{m}$ when one of the shunt plates is in the position of $\mathrm{D}=13 \mathrm{~mm}$ while the other is $\mathrm{D}=40 \mathrm{~mm}$. No significant deformation in the horizontal axis, $d B_{y} / d x$, is found. When the asymmetry needs to be avoided, one may set the same positions D for both the plates while keeping the total variable range, 3.9\%.

\section{B. Leakage field}

After the temperature-dependence compensation and adjustment of fringe field cancellation, three-dimensional magnetic field measurement was performed with a temperature compensated Hall probe, (three-axis probe MMZ2508-UH, three-channel Hall effect gaussmeter Model 460, Lake Shore Cryotronics, Inc.).

Figure 14 shows the transverse distribution of magnetic flux density, $B_{y}$, at the longitudinal center. The simulated fringe field without a septum (black in Fig. 14) shows no significant reduction compared with the gap field (1.2 T), while that with a septum (blue) is suppressed to below $10^{-3} \mathrm{~T}$. The measured fringe field with the septum is consistent with that expected by the simulation. The residual leakage field can be shielded below the geomagnetic intensity by surrounding the beam chamber with a mu metal sheet. We assume the distance between the incident beam and the septum plate is $15 \mathrm{~mm}$. The stored beam on the bump orbit can be nearly $30 \mathrm{~mm}$ from the incident beam at the downstream end of the dc septum.

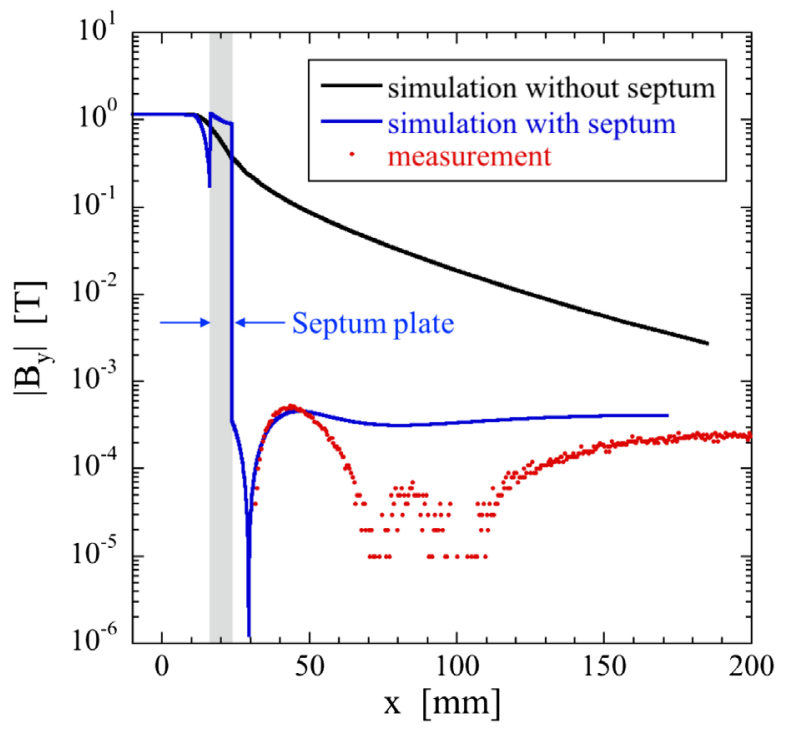

FIG. 14. Transverse distribution of magnetic flux density at the longitudinal center $(\mathrm{z}=0 \mathrm{~mm})$ of the dc septum. Black and blue lines represent the simulated distribution without and with the septum plate, respectively. Red dots represent the measured data with the septum plate. The origin of horizontal axis is defined at the incident beam position. The septum plate occupies the position between $\mathrm{x}=15$ and $22 \mathrm{~mm}$.

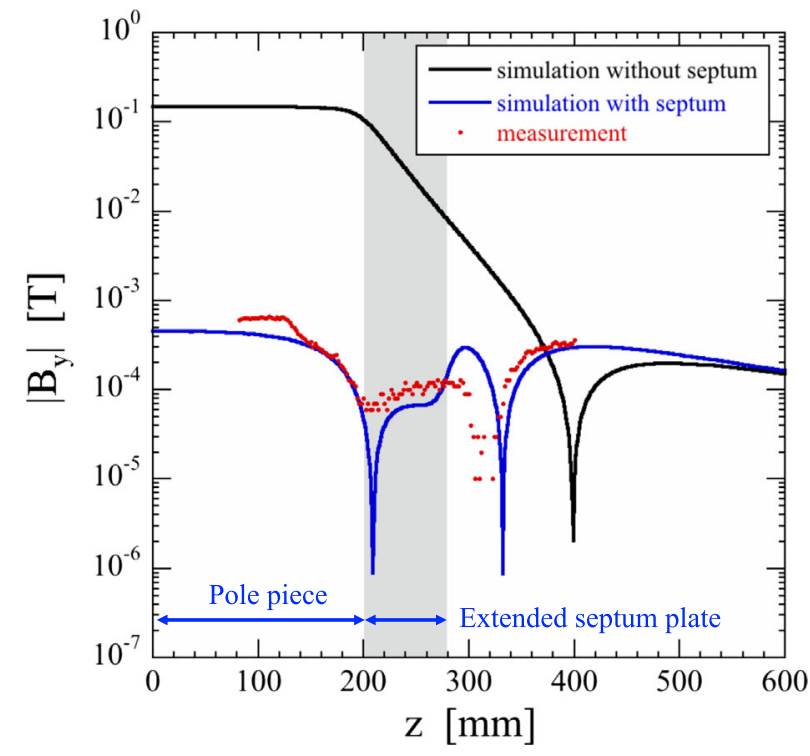

FIG. 15. Longitudinal distribution of magnetic flux density at $\mathrm{x}=47 \mathrm{~mm}$. Black and blue lines represent the simulated distribution without and with the septum plate, respectively. Red dots represent the measured data with the septum plate.

In the longitudinal direction, the field strength is also significantly suppressed, as shown in Fig. 15, and again the measured field with the septum is well suppressed as designed using the simulation.

\section{REDUCTION IN SEPTUM THICKNESS}

Aiming at further reduction of the injection amplitude for the future, we here discuss a possibility of reducing the septum thickness even further. One of the solutions is the multilayering of the septum plate. The advantage of using multilayer shielding is well known [21].

We employed a double-septum structure with a nonmagnetic gap in between as shown in Fig. 16. As an example, an aluminum plate can work as the nonmagnetic gap. In Fig. 16, the thickness of the septum plate on the injection beam side (left in Fig. 16) has a thickness of

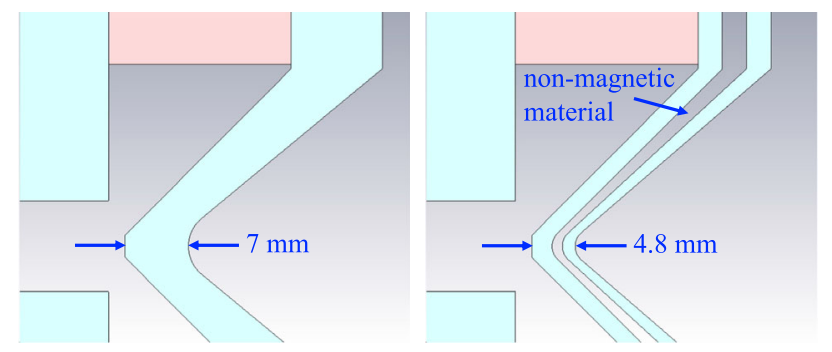

FIG. 16. Comparison between single- and double-septum structures. (Left) Original shape with the thickness of $7 \mathrm{~mm}$ at the median plane. (Right) Double layer of the iron with nonmagnetic gap between them. The thickness is reduced by $2.2 \mathrm{~mm}$ compared to the original shape. 


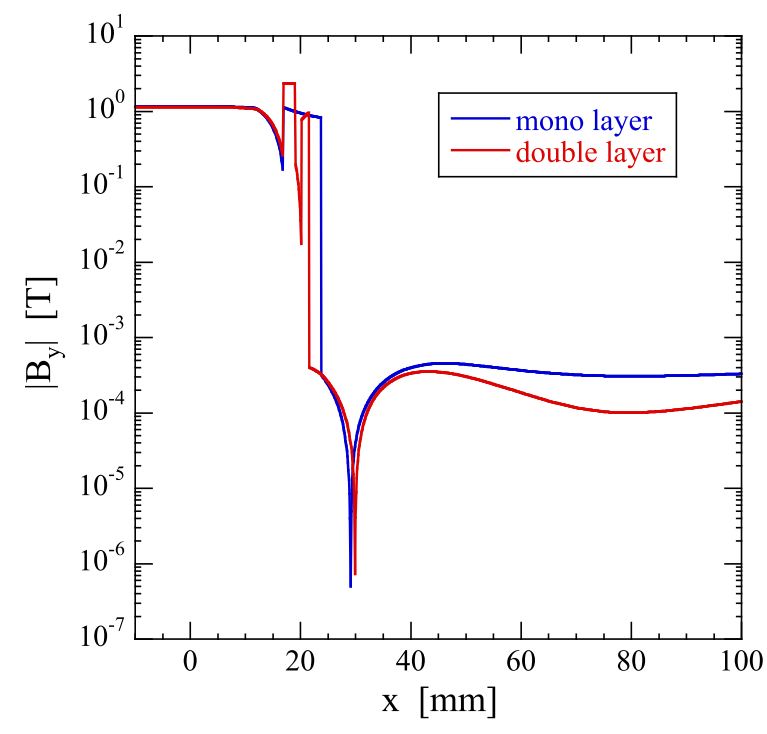

FIG. 17. Transverse distribution of magnetic flux density at the longitudinal center $(\mathrm{z}=0 \mathrm{~mm})$ of the $\mathrm{dc}$ septum. Blue line represents the simulated distribution for the original 7-mm-thick mono layer and red line represents that for 4.8-mm-thick double layer septum.

$2.2 \mathrm{~mm}$, while that on the stored beam is $1.4 \mathrm{~mm}$. The gap in between is $1.2 \mathrm{~mm}$, so that the overall septum thickness is $4.8 \mathrm{~mm}$, which is as much as $2.2 \mathrm{~mm}$ thinner than the original case of the single-septum structure. The transverse distribution of magnetic flux density at the longitudinal center $(\mathrm{z}=0 \mathrm{~mm})$ of the dc septum is plotted in Fig. 17. Thus, it is verified that the double-septum structure enables us to significantly reduce the septum thickness while keeping the same level of the leakage field. The thickness will be reduced by the increase in a number of layers even further as far as it is reasonably feasible in practical fabrications of the magnet.

\section{CONCLUSION}

A PM-based septum magnet was developed for the injection section of next-generation light sources. The fringe magnetic field on the orbit of the stored beam was shielded by a 7-mm thick septum plate with counter-field PMs. The gap field strength can be varied by displacing the shunt plate. Issues unique to a PM, such as temperature characteristic and demagnetization, were also solved. Furthermore, we numerically demonstrated that the septum thickness can be reduced further by multilayering the septum plate. In our case, the overall thickness was reduced by $2.2 \mathrm{~mm}$ with the doubleseptum structure.

The permanent magnet has drawn attention as a viable candidate in the development of next-generation light sources and related fields. It is reasonable to fabricate injection dc magnets, which normally consume large amounts of power, using permanent magnets, in regards to power consumption reduction, power supply failure prevention, etc. Future developments based on this study are expected not only to help accelerators reduce their power consumption, but also engender new possibilities for choosing even higher packing factor lattices and other challenging accelerator designs with ease.

\section{ACKNOWLEDGMENTS}

This work was supported by JST Next Generation Accelerator Technology Development Program and the RIKEN SPring-8 Center (RSC). The authors would like to thank H. Tanaka for directing the development program of the PM-based septum magnet. One of the authors (T. W.) would like to acknowledge J. Chavanne for overall discussion on magnets.

[1] H. Tanaka, T. Ishikawa, S. Goto, S. Takano, T. Watanabe, and M. Yabashi, SPring-8 upgrade project, in Proceedings of the 7th International Particle Accelerator Conference, Busan, Korea, 2016 (JACoW, Geneva, 2016), pp. 2867-2870.

[2] P. Raimondi, ESRF-EBS: The extremely brilliance source project, Synchrotron Radiat. News 29, 8 (2016).

[3] L. Liu, F. H. de Sá, and X. R. Resende, A new optics for sirius, in Proceedings of the 7th International Particle Accelerator Conference, Busan, Korea, 2016 (JACoW, Geneva, 2016), pp. 3413-3416.

[4] H. D. Glass, B. C. Brown, G. W. Foster, W. B. Fowler, J. E. Haggard, D. J. Harding, G. P. Jackson, M. P. May, T. H. Nicol, J. F. Ostiguy, P. Schlabach, G. A. Smith, and J. T. Volk, Permanent dipole magnets for the $8 \mathrm{GeV}$ transfer line at FNAL, in Proceedings of the Particle Accelerator Conference, Vancouver, BC, Canada, 1997 (IEEE, New York, 1997), Conf 97-3257.

[5] Y. Iwashita, Y. Tajima, M. Ichikawa, S. Nakamura, T. Ino, S. Muto, and H. M. Shimizu, Variable permanent magnet sextupole lens for focusing of pulsed cold neutrons, Nucl. Instrum. Methods Phys. Res., Sect. A 586, 73 (2008).

[6] Y. Iwashita, M. Yamada, S. Ushijima, Y. Fuwa, Y. Nasu, H. Tongu, M. Masuzawa, and H. M. Shimizu, Variable permanent magnet multipoles, IEEE Trans. Appl. Supercond. 22, 4000905 (2012).

[7] C. Benabderrahmane, J. C. Biasci, J. F. Bouteille, J. Chavanne, L. Eybert, L. Goirand, G. Le Bec, L. Lefebvre, S. M. Luizzo, D. Martin, C. Penel, P. Raimondi, J. L. Revol, F. Villar, and S. White, Status of the ESRF-EBS magnets, in Proceedings of the 9th International Particle Accelerator Conference, Vancouver, BC, Canada, 2018 (JACoW, Geneva, 2018), pp. 2648-2651.

[8] P. N'gotta, G. Le Bec, and J. Chavanne, Hybrid high gradient permanent magnet quadrupole, Phys. Rev. Accel. Beams 19, 122401 (2016).

[9] J. Citadini, L. N. P. Vilela, R. Basilio, and M. Potye, Siriusdetails of the new $3.2 \mathrm{~T}$ permanent magnet superbend, IEEE Trans. Appl. Supercond. 28, 4101104 (2018).

[10] Y. Iwashita and A. Noda, Massless septum with hybrid magnet, in Proceedings of 6th European Particle 
Accelerator Conference, Stockholm, Sweden, 1998 (IOP, London, 1998), pp. 2109-2110.

[11] T. Kawakubo, E. Nakamura, M. Numajiri, M. Aoki, T. Hisamura, and E. Sugiyama, Permanent magnet generating high and variable septum magnetic field and its deterioration by radiation, in Proceedings of 9th European Particle Accelerator Conference, Lucerne, Switzerland, 2004 (EPS-AG, Lucerne, 2004), pp. 1696-1698 [http:// accelconf.web.cern.ch/AccelConf/e04/].

[12] T. Watanabe, T. Taniuchi, S. Takano, T. Aoki, and K. Fukami, Permanent magnet based dipole magnets for next generation light sources, Phys. Rev. Accel. Beams 20, 072401 (2017).

[13] S. Takano, K. Fukami, C. Kondo, M. Masaki, M. Oishi, M. Shoji, K. Tamura, T. Taniuchi, K. Yanagida, T. Watanabe, K. Hamato, J. Kataoka, K. Kusano, K. Ogata, Y. Saito, H. Akikawa, and K. Sato, Renovation of off-axis beam injection scheme for next-generation photon sources, in Proceedings of the 10th International Particle Accelerator Conference, Melbourne, Australia, 2019 (JACoW, Geneva, 2019), pp. 2318-2321.

[14] TOKIN Corporation, LM-32SH, https://www.tokin.com/ english/product/pdf_dl/permanentmagnets.pdf.
[15] T. Bizen, R. Kinjo, T. Hasegawa, A. Kagamihata, Y. Kida, T. Seike, T. Watanabe, T. Hara, T. Itoga, Y. Asano, and T. Tanaka, Radiation-induced magnetization reversal causing a large flux loss in undulator permanent magnets, Sci. Rep. 6, 37937 (2016).

[16] Dassault Systèmes, CST Studio Suite, https://www.3ds .com/products-services/simulia/products/cst-studio-suite/.

[17] Japanese Industrial Standards, JIS G 3101, Rolled steels for general structure.

[18] J. Chavanne, P. Elleaume, and P. V. Vaerenbergh, The ESRF insertion devices, J. Synchrotron Radiat. 5, 196 (1998).

[19] Hitachi Metals Neomaterial, Ltd., MS-2, http://www .hitachi-metals-neomaterial.co.jp/english/product/liv/08 .html.

[20] T. Bizen, Y. Asano, T. Hara, X. Marechal, T. Seike, T. Tanaka, H. S. Lee, D. E. Kim, C. W. Chung, and H. Kitamura, Baking effect for $\mathrm{NdFeB}$ magnets against demagnetization induced by high-energy electrons, Nucl. Instrum. Methods Phys. Res., Sect. A 574, 401 (2007).

[21] T. J. Sumner, J. M. Pendlebury, and K. F. Smith, Conventional magnetic shielding, J. Phys. D 20, 1095 (1987). 